\title{
Giant Aplysia Neuron R2 Has Distal Dendrites: Evidence for Protein Sorting and a Second Spike Initiation Site
}

\author{
Richard T. Ambron, ${ }^{2}$ Stephen G. Rayport, ${ }^{1,2,3}$ and Joanne Babiarz ${ }^{2}$ \\ ${ }^{1}$ Center for Neurobiology and Behavior, ${ }^{2}$ Departments of Anatomy and Cell Biology, and ${ }^{3}$ Psychiatry, Columbia University \\ College of Physicians and Surgeons and the New York State Psychiatric Institute, New York, New York 10032
}

\begin{abstract}
Because of its anatomy, the neuron R2 of Aplysia has been used to study how proteins are distributed to their appropriate destinations within the cell. The $R 2$ cell body resides in the abdominal ganglion, while its axons terminate on glands in the skin. Using intracellular injection of HRP and intraxonal recordings, we found that $\mathbf{R 2}$ has a dendritic (receptive) arborization in the pleural ganglion. The structure of these dendrites was examined after injecting the soma with ${ }^{3} \mathrm{H}-\mathrm{L}-$ fucose, thereby labeling glycoproteins that are transported to all regions of the cell. Light- and electron-microscope autoradiography show that the openings to the dendrites are not on the periphery, but are suspended inside the axon by glial cell infoldings. All of the organelles seen in the axon are found in the dendrites, including 2 types of vesicles. Neither the axon nor the dendrites contain ribosomes. Thus, R2 has 3 functionally distinct regions-cell body, dendrites, presynaptic terminals-that are separated from each other by at least $4 \mathrm{~cm}$. This implies that pre- and postsynaptic proteins made in the cell body are transported along the axon to the pleural ganglion, where they are sorted. To investigate this idea, we exposed the abdominal ganglion to ${ }^{35} S$-methionine to label R2's proteins. Analyses by SDS-PAGE of the rapidly transported labeled proteins from R2 consistently showed a $78 \mathrm{kDa}$ band that accumulated in the pleural ganglion and did not move into the peripheral nerves. This then is a putative dendritic constituent.
\end{abstract}

Neurons are highly differentiated cells consisting of distinct regions that differ in structure and function. Such regional distinctions reflect differences in macromolecular composition. Because the cell body is the site of synthesis of macromolecules, mechanisms must exist to sort molecules for transport to their appropriate location in the cell. The routing of specific macromolecules occurs in all polarized cells and has been investigated in a variety of epithelial tissues (for a review, see Simons and Fuller, 1985). Characterization, at the molecular level, of the processes involved in sorting is important for an under-

\footnotetext{
Received Feb. 9, 1987; revised July 21, 1987; accepted Aug. 25, 1987.

We thank Drs. C. Bailey, D. Goldberg, I. Kupfermann, and S. Schacher for their comments on an earlier version of the paper, and Ms. Marilyn Smedman for expert technical help. Supported by National Institutes of Health Research Grant NS-22150 and a Hirschl Career Development Award to R.T.A., M.D.Ph.D. Program Fellowship Grant GM-07367 to S.G.R., Grant GM-23540 to E.R. Kandel and NIH Animal Research Grant RR-01215 to the mariculture facility, Marinc Biological Laboratory, Woods Hole, MA.

Correspondence should be addressed to Richard Ambron, Ph.D., Department of Anatomy and Cell Biology, College of Physicians and Surgeons, Columbia University, 630 W. 168 th St., New York, NY 10032.

Copyright (C) 1988 Society for Neuroscience $0270-6474 / 88 / 020722-11 \$ 02.00 / 0$
}

standing of how cells are maintained in a functionally differentiated state.

Neurons offer an advantage for such studies, since their differentiated regions, such as the presynaptic terminal, can be at a great distance from the cell body. This spatial segregation thereby facilitates the identification of sorted constituents. R2, the giant neuron of Aplysia, is an especially advantageous preparation in this regard. The $\mathrm{R} 2$ cell body resides in the abdominal ganglion (Fig. 1); its main axon gives off a series of dendrites and then exits from the ganglion to course unbranched within the right connective to the pleural ganglion in the head. There the axon bifurcates, ultimately sending branches through ipsilateral and contralateral peripheral nerves to the body wall. R2 axons terminate on mucous glands in the skin (Rayport et al., 1983). In the adult, some of the endings may be over $10 \mathrm{~cm}$ from the cell body.

Two mechanisms have been found that contribute to establishing intracellular polarity in R2. The first is selective transport, whereby only certain proteins (Ambron et al., 1985; Drake et al., 1985) and glycoproteins (Ambron, 1981) made in the cell body are exported into the axon for rapid transport to the periphery. The second is the posttranslational modification of proteins in the axon (Ambron and Treistman, 1977). Both mechanisms were thought to be involved in segregating presynaptic constituents.

In this report we show that $\mathrm{R} 2$ has a dendritic (receptive) arborization arising from the axon in the pleural ganglion, a distance of some $4 \mathrm{~cm}$ from the cell soma. The presence of these dendrites creates a logistic problem for the cell. Since the dendrites contain neurotransmitter receptors that presumably are not present at R2 terminals in the skin, the rapidly transported fraction must consist of both pre- and postsynaptic constituents. Once these constituents reach the pleural ganglion, they must be sorted from each other. Yet, unlike the situation in other polarized cells, this sorting occurs at a great distance from the biosynthetic site.

The finding of distal dendrites also complicates our understanding of how $\mathrm{R} 2$ regulates mucus release, but may explain the bidirectional action potentials seen in the $\mathrm{R} 2$ right connective axon in frecly moving animals (Ebcrly and Pinsker, 1979).

\section{Materials and Methods}

Intrasomatic injection of HRP. The distance between the cell body of R2 and the pleural ganglion was minimized by using late juvenile animals. These animals, raised at the Marine Biological Laboratory (Woods Hole, MA), are about $2 \mathrm{~cm}$ long and weigh about $1 \mathrm{gm}$. They were anesthetized by injection of isotonic $\mathrm{MgCl}_{2}$ into the body cavity. The central nervous system was removed intact and the $\mathrm{R} 2$ cell soma was 
pressure-injected with $10 \%$ HRP (Sigma Chemicals, type VI) in $0.1 \mathrm{~N}$ $\mathrm{NaOH}$ (Rayport et al., 1983). After injection, the nervous system was maintained in artificial seawater (Eisenstadt et al., 1973) for $48 \mathrm{hr}$ at $15^{\circ} \mathrm{C}$, fixed with $6 \%$ glutaraldehyde in $S$-collidine buffer, reacted with diaminobenzidine, dehydrated, and embedded in Permount (Bailey et al., 1981). Tissue was examined by Nomarski differential interference optics and by electron microscopy.

Electrophysiology. The remaining experiments were performed using adult Aplysia (150 gm; Marine Specimens, Venice, CA). Animals were anesthetized, the central nervous system was removed, and the abdominal and pleural ganglia were pinned in a Sylgard-coated dish (DowCorning). After desheathing to expose the R2 cell body and distal axon, respectively, a suction electrode was placed on pedal nerve P8. Using conventional neurophysiological techniques and fine, single-barreled electrodes, we impaled the R2 soma and the axon in the pleural ganglion. Electrodes for axonal recordings were made using a Brown-Flaming micropipette puller (Sutta Instruments). Simultaneous and stable recordings with full resting potential and spike amplitude were reliably obtained (see also Rayport and Kandel, 1980). To test the response of the dendrites to exogenous neurotransmitters, the right connective was threaded through a Vaseline seal so that soma and distal dendrites could be perfused separately. Neurotransmitters, mixed in saline immediately prior to use, were applied to the bath directly above the desheathed abdominal or pleural ganglion.

Metabolic labeling of $R 2$ with ${ }^{3} \mathrm{II}-\mathrm{fucose}$ and ${ }^{35} \mathrm{~S}-$ methionine. ${ }^{3} \mathrm{H}-\mathrm{L}-$ Fucose (New England Nuclear, $24 \mathrm{Ci} / \mathrm{mmol}$ ) was injected into R2's cell body in the isolated central nervous system (Ambron et al., 1974). The tissue was maintained for $50 \mathrm{hr}$ and was then processed for light and electron microscope autoradiography (Ambron et al., 1979; Bailey et al., 1981). For serial reconstructions, sequential $90 \mathrm{~nm}$ sections were cut through the pleural ganglion in the region of R2's dendrites. They were examined and electron micrographs recorded using a Phillips 301 electron microscope.

Radiolabeled proteins, rapidly transported to the pleural ganglion and peripheral nerves, were obtained by placing the abdominal ganglion in a $0.3 \mathrm{ml}$ chamber separated from the attached nervous system by a Vaseline seal around the right connective. The ganglion was exposed to $250-400 \mu \mathrm{Ci}$ of ${ }^{35} S$-methionine (Amersham; $>1000 \mathrm{Ci} / \mathrm{mmol}$ ) for $5 \mathrm{hr}$. The remaining portion of the connective and the ganglia in the outer bath were bathed in artificial seawater containing $5 \mathrm{~mm}$ methionine (Ambron et al., 1985). After a $50 \mathrm{hr}$ chase, the R2 cell body, removed from the ganglion, the pleural ganglion containing the dendrites, and the peripheral nerves containing R2's axons were collected separately. Each sample was homogenized in a ground-glass grinder in SDS buffer, heated at $70^{\circ} \mathrm{C}$ for $15 \mathrm{~min}$ and, after centrifugation, the extracts were separated on a 5-15\% linear gradient slab gel (Laemmli, 1970). The gel was fixed, infiltrated with sodium salicylate, and dried. Fluorography was carried out according to Bonner and Lasky (1974) using flashed Kodak X-Omat film. Lanes of fluorographs were scanned using a JoyceLoebel microdensitometer. Stained molecular-weight standards were obtained from Bethesda Research Laboratories (Gaithersburg, MD).

\section{Results}

\section{$R 2$ has dendrites in the pleural ganglion}

During earlier studies, recordings from the $\mathrm{R} 2$ axon revealed apparent chemical excitatory synaptic input at a distance from the cell body (Rayport, 1981). The morphological basis for this synaptic input was found by tracing HRP that had been injected into R2's cell body. Within $48 \mathrm{hr}$, reaction product had entered a series of evaginations from R2's axon in the pleural ganglion (Fig. 2). These stuctures were especially prominent in the region just before the axon bifurcation. They ramified extensively and extended into the neuropil region of the ganglion. Structures with a similar appearance and distribution are typically found near the cell body of Aplysia neurons (Winlow and Kandel, 1976), and R2 has such an arborization adjacent to its cell soma in the abdominal ganglion (Thompson et al., 1976). We will refer to these structures as "dendrites," meaning only that they are postsynaptic regions of $\mathrm{R} 2$.

To show directly that the R2 axon in the pleural ganglion receives synaptic input, we placed an clcctrode in the axon at

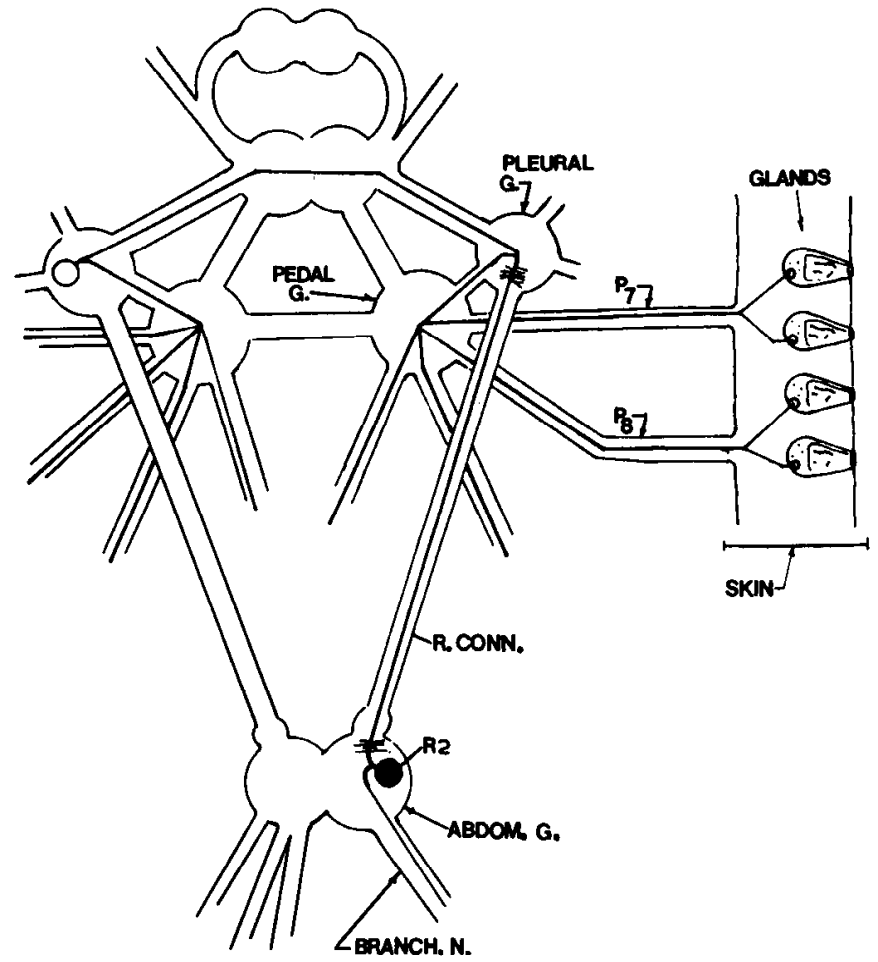

Figure 1. Schematic showing the distribution of the axon of R2. The $\mathrm{R} 2$ cell body resides in the abdominal ganglion. Its axons travel within ipsilateral and contralateral peripheral nerves to terminate on unicellular mucous glands in the skin. Nerves $P 7$ and $P 8$ contain large branches of the R2 axon and were collected in experiments to examine R2's proteins that were rapidly transported to presynaptic terminals. Two sets of dendrites are shown; one set evaginates from the axon proximal to the cell body, the other from the axon as it courses through the pleural ganglion. Nerve P8 was used for electrophysiological stimulation of afferents to R2's pleural dendrites.

the site of the presumed dendrites (Fig. 3). Identification of the axon was confirmed by intrasomatic stimulation of $\mathrm{R} 2$, which reliably elicited an axon spike at fixed latency (Fig. $3 A$ ). To activate cells that might project to $\mathrm{R} 2$ we stimulated peripheral nerve P8. This nerve contains an axon of R2 (as do all other nerves to the body wall), and low-voltage stimulation consistently evoked antidromic potentials, first in the axon and then in the soma. Raising the voltage, however, activated smaller fibers in the nerve and evoked polysynaptic input in the intraganglionic axon and, after a delay, in the R2 soma. To see this input more clearly, we collided an orthodromic R2 spike with the antidromically stimulated spike: this physiological subtraction of R2's antidromic response clearly revealed the polysynaptic input (Fig. 3).

\section{Common transmitters do not elicit axonal polarizations}

We attempted to identify the neurotransmitter released by the neurons presynaptic to $\mathrm{R} 2$. In the desheathed preparation used for the axonal recordings, we applied acetylcholine, serotonin, octopamine, and histamine to see if they could mimic the excitatory input onto the R2 axon. Although each transmitter elicited a response when added in the region of the cell soma, assuring an adequate drug concentration, recordings from the axon in the pleural ganglion revealed no effect. 


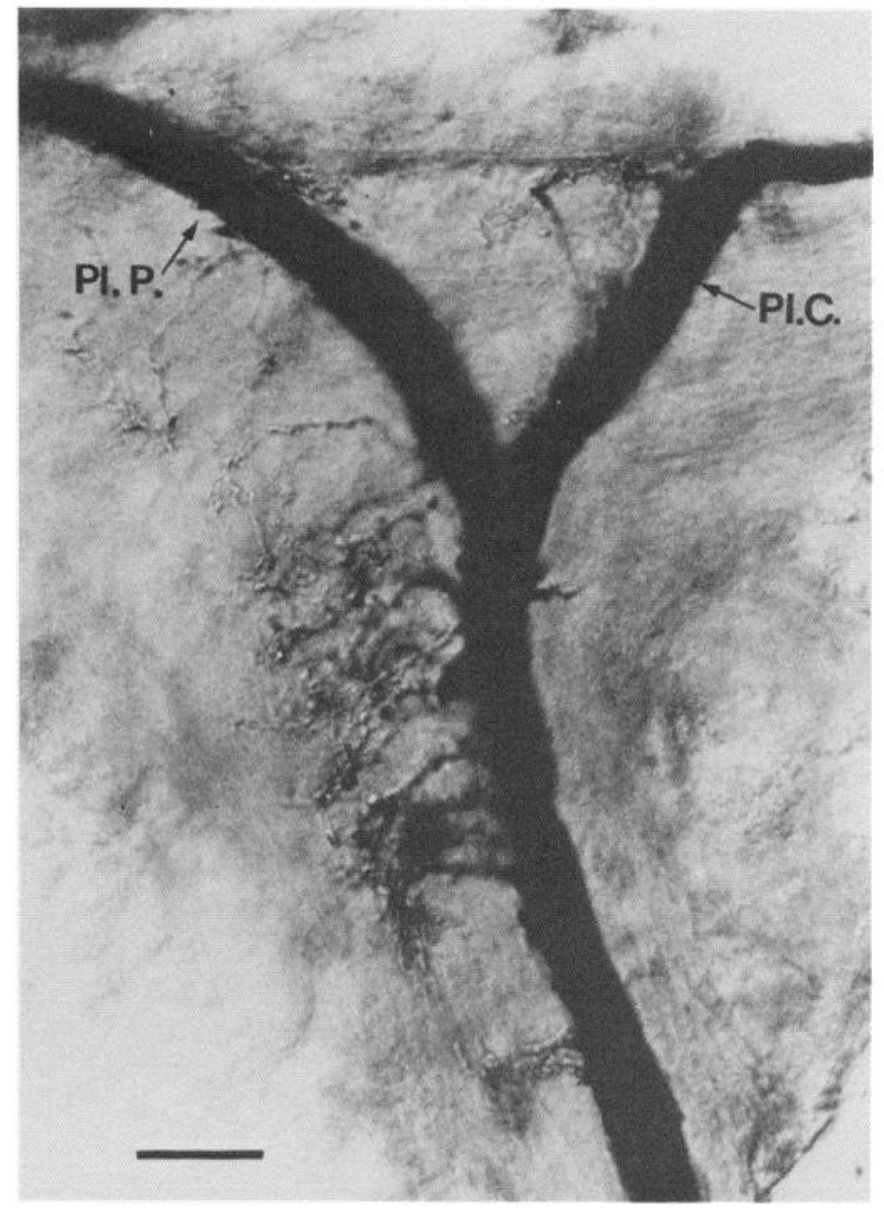

Figure 2. HRP-filled dendrites in the pleural ganglion. R2 was injected with HRP in the isolated central nervous system and $48 \mathrm{hr}$ later the tissue was processed to visualize the HRP. R2's axon in the pleural ganglion is seen giving rise to a pleurocerebral $(P l . C$. $)$ branch projecting to the cerebral ganglion and a pleuropedal (Pl.P.) branch going to the pedal ganglion (see Fig. 1). Proximal to the bifurcation, numerous dendritic evaginations are evident. Calibration, $50 \mathrm{~nm}$.

\section{Morphology of R2's distal dendrites}

Origin of the dendrites from the axon. The detailed structure of the dendrites was studied by light- and electron-microscope autoradiography $50 \mathrm{hr}$ after injecting R2's cell body with ${ }^{3} \mathrm{H}$ fucose. This sugar is incorporated into several membrane glycoproteins that are rapidly transported along R2's axon to the periphery. The fact that all of the radiolabeled glycoproteins are restricted to $\mathrm{R} 2$ and its processes makes this a convenient technique for identifying the various regions of a neuron (Thompson et al., 1976). We traced the R2 axon in sections through the pleural ganglion and observed that ${ }^{3} \mathrm{H}$-glycoproteins had entered R2's dendrites (Fig. 4A). An unexpected finding was the presence of discrete areas of very high grain density inside the axon (Fig. $4, B, C$ ). Electron microscopy showed that these areas are enclosed by a double membrane, one contributed by the invaginating axolemma, the other by the glial cells that envelope the axon and penetrate deep into the axoplasm (Fig. 4D).

The fact that transverse sections through the axon also present a transverse view of the dendrites indicates that these structures are arranged as a tube within a tube. Sequential sections obtained from various regions along the axon showed that the dendrites can course within the axon for several hundred microns. To find the origin of the dendrites, we sectioned the pleural ganglion parallel to the long axis of R2's axon. Since we had already identified the axon by the presence of grains, the sections were not processed for autoradiography. Two openings were found (Fig. 5). As expected, they were not on the periphery, but were suspended inside the axon.

On the basis of studies of the electron micrographs and analysis of the origin of the 2 membranes that surround the dendrites (Fig. 4), we constructed a diagram showing the relationship between axon, dendrite, and glial cells (Fig. 6). The infolding of the glial membrane presumably serves to stabilize the dendrite within the axon. We wish to point out that we have examined only a small portion of the R2 axon in the pleural ganglion, and it is possible that conventional dendritic evaginations exist as well.

Ultrastructure of R2's dendrites. The dendrites, identified by their content of silver grains, were followed into the neuropil of

Figure 3. Synaptic input to R2. Intracellular recordings were obtained from the $\mathrm{R} 2$ cell body in the abdominal ganglion and from the R2 axon in the right pleural ganglion. $A$, An orthograde spike was elicited in the R2 soma by intrasomatic depolarizing current, confirming the position of the axonal electrode, and blocking the retrograde spike that would otherwise have been initiated by stimulation $(S)$ of pedal nerve P8. A large-population EPSP is evident following the nerve stimulus which, with greater latency, is also seen in the cell body. $B$, Nerve stimulation is applied more closely following the intracellularly elicited spike, showing that the population EPSPs move in correspondence with the orthograde spike. Additionally, the synaptic input onto the $\mathrm{R} 2$ cell soma is sufficient to elicit a spontaneous action potential, which demonstrates the normal configuration of the axonal spike afterpolarization in the absence of synaptic input in the pleural ganglion.

A

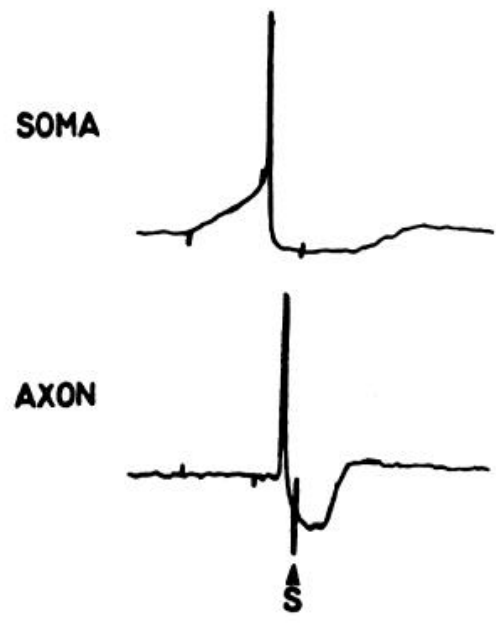

B

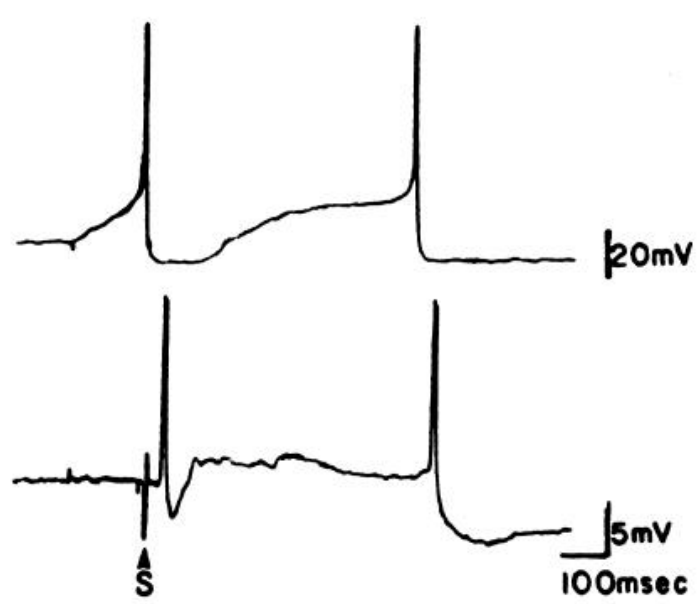



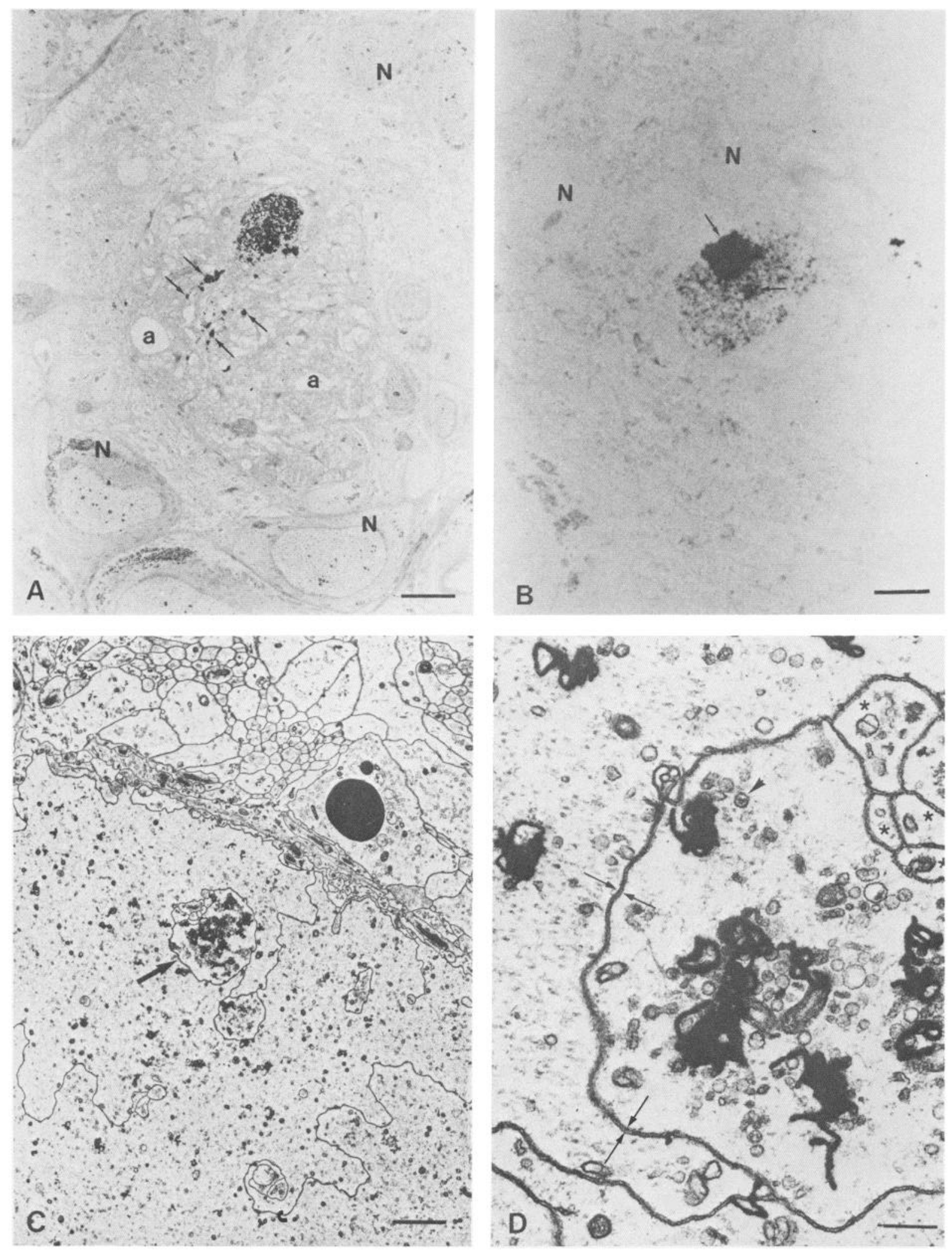

Figure 4. Examination of R2's dendrites by autoradiography $48 \mathrm{hr}$ after intrasomatic injection of ${ }^{3} \mathrm{H}$-fucose. Transverse sections through the pleural ganglion are shown. $A$, Light micrograph of a stained section showing grains in the $\mathrm{R} 2$ axon and dendritic arborization (arrows); $a$, axons of other neurons; $N$, pleural ganglion neurons. Calibration, $25 \mu \mathrm{m}$. $B$, Adjacent section, unstained, showing 2 discrete areas of very high grain density within R2's axon (arrows). Calibration, $25 \mu \mathrm{m}$. C. Electron-microscope autoradiograph showing one of the highly labeled areas within the axon. Other axons and the glial cells that surround R2's axon are seen in the upper part of the micrograph. Grains are distributed throughout the $\mathrm{R} 2$ axon, but are concentrated over organelles in a membrane-bound tube. The tube is a cross section through one of the dendrites. Calibration, $1 \mu \mathrm{m}$. $D$, At higher magnification, a region of the labeled dendrite shown in $C$ is seen to contain lucent and compound vesicles (arrowhead) and to be surrounded by a double layer of membrane (arrows). Glial cell infoldings (asterisks) found on the periphery do not contain grains. Calibration, $0.3 \mu \mathrm{m}$. 


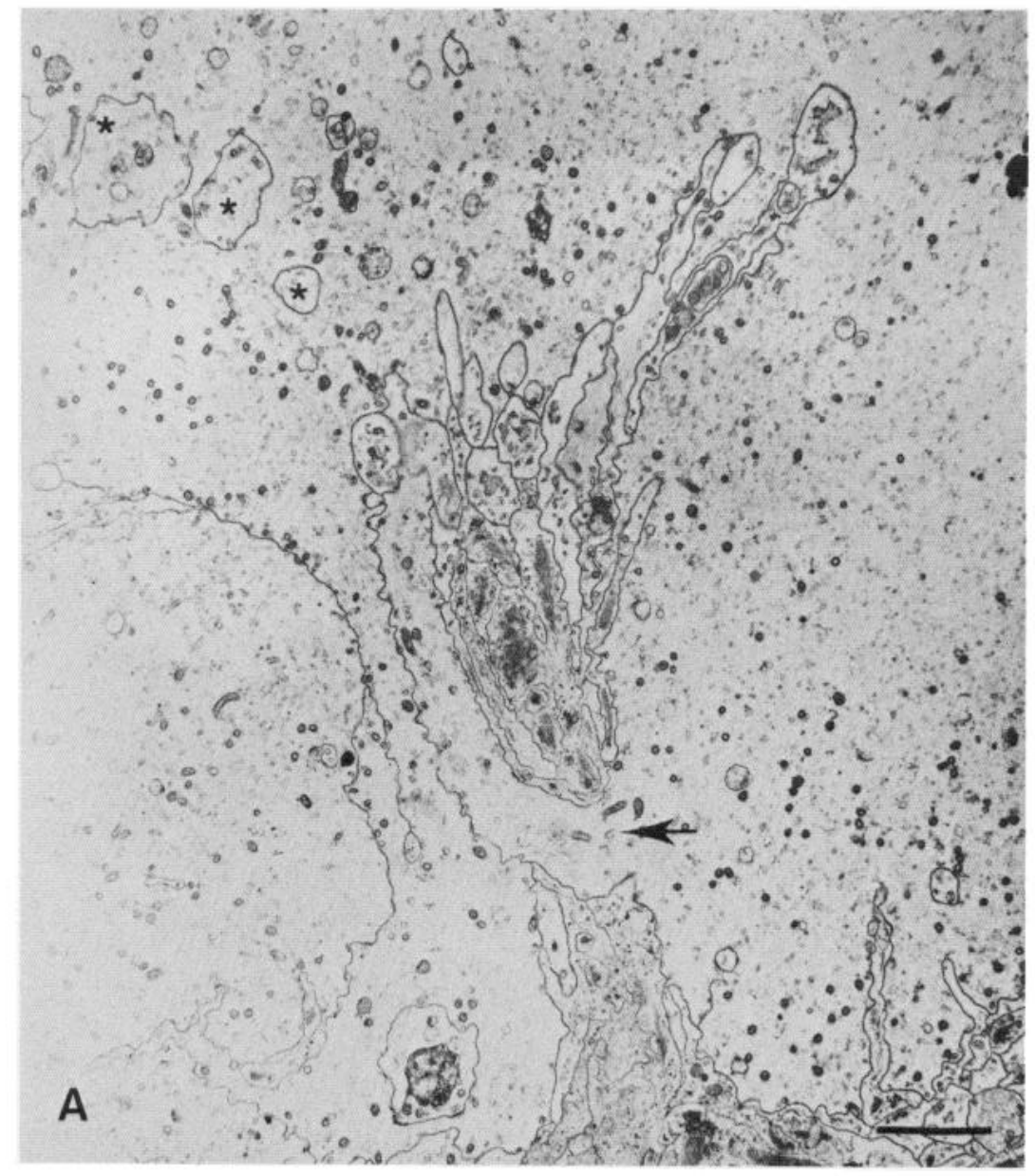

Figure 5. Origin of the dendrites within the axon. The pleural ganglion was sectioned parallel to the direction of the $\mathrm{R} 2$ axon. The openings to the dendrites are indicated by arrows. Each entrance is surrounded by glial cell processes. $A$, Examination of adjacent sections shows that the dendrite courses through the axon prior to exiting (asterisks). Calibration, $20 \mu \mathrm{m}$. $B$, Vesicles and other organelles are seen entering the dendrite. Calibration, $20 \mu \mathrm{m}$.

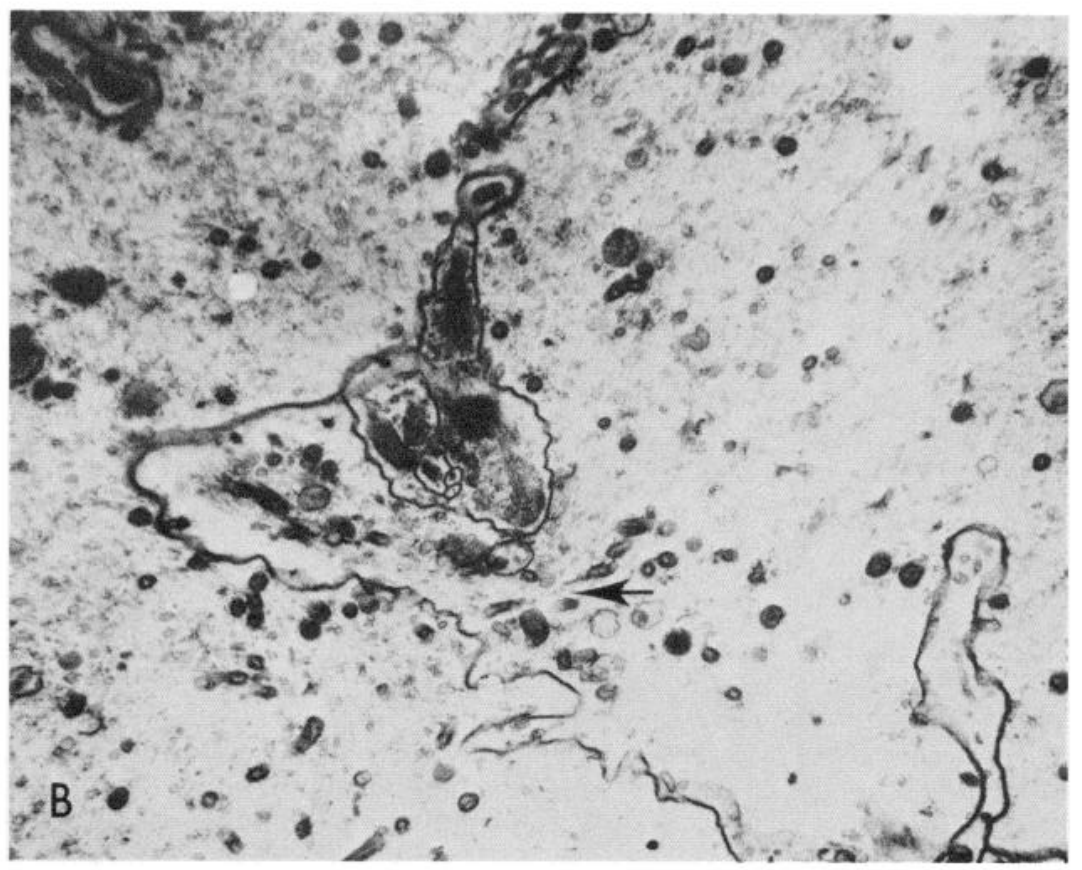

the pleural ganglion (Fig. 7A). They ramified extensively and came into contact with processes of many different neurons. Some of the contacts showed accumulations of vesicles and had other characteristics of Aplysia synapses (for example, see Fig.
$7 B$ ). It will require serial section reconstruction to be sure that they are actually presynaptic endings onto $\mathrm{R} 2$, however. We also observed fingerlike structures of unknown origin projecting into R2's processes (Fig. 7C). 


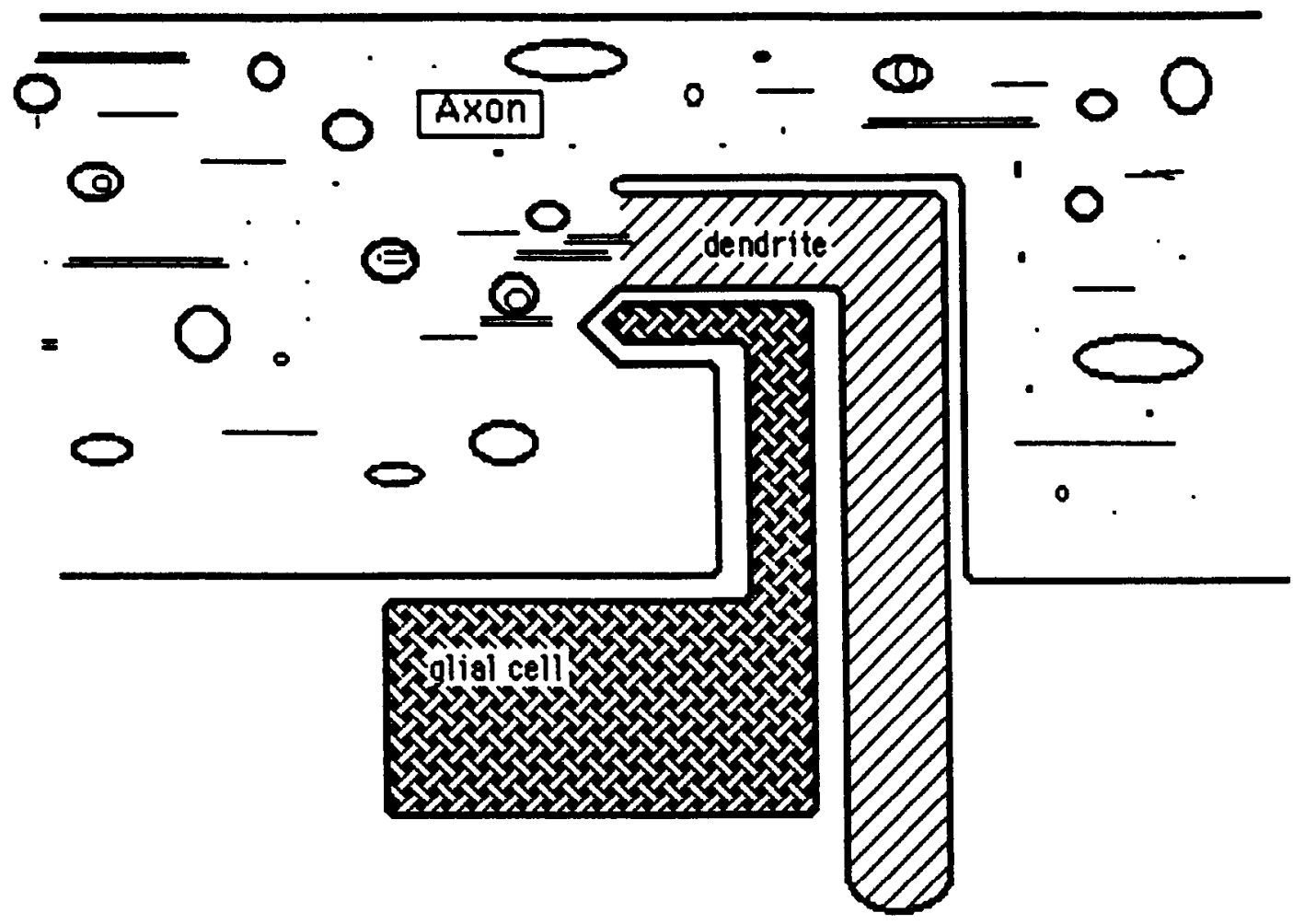

Figure 6. Schematic of the origin of $\mathbf{R} 2$ dendrites. The relationship between the glial cells and the dendrites is shown in longitudinal section. The scheme is not drawn to scale. Note that the invaginated membrane of the axon envelops both the origin of the dendrite and the glial cell process. A cross section through the opening would correspond to the view shown in Figure $4 D$.

Organelles that are transported into the dendrites seem to be representative of those found in the axon. Previous studies of R2 described 2 kinds of vesicles: lucent, $80 \mathrm{~nm}$ in diameter, and compound, $90 \mathrm{~nm}$ in diameter (Thompson et al., 1976; Ambron et al., 1979). The latter consist of small vesicles enclosed within larger ones. We found both vesicle types in the dendrites (Fig. 4D), as well as mitochondria and various irregularly shaped membrane profiles. As with the R2 axon, there were no ribosomes in the dendrites.

\section{Proteins transported into the dendrites}

Since the R2 dendrites receive input from other neurons, they must contain neurotransmitter receptors, etc., that would not be expected at R2's presynaptic terminals in the periphery. To identify postsynaptic constituents, we exposed the abdominal ganglion to ${ }^{35} S$-methionine for $5 \mathrm{hr}$, followed by a $50 \mathrm{hr}$ chase. We know from previous biochemical and autoradiographic studies that, under these conditions, radiolabeled protein is rapidly transported along the right connective. Most of the transported protein is made by neurons $\mathrm{R} 2$ and $\mathrm{R} 1$. By $50 \mathrm{hr}$, protein has accumulated in the $\mathrm{R} 2$ dendrites and has been distributed along the peripheral nerves that contain $\mathrm{R} 2$ axons (Ambron et al., 1985). The constituents that accumulate in the dendrites were identified by using SDS-PAGE to compare the labeled polypeptides in the distal right connective and pleural ganglion with those in the peripheral nerves.

In 6 experiments, we consistently observed a $78 \mathrm{kDa}$ band that was prominent in the connective and ganglion, but that never appeared in the periphery (Fig. 8). This is the distribution that would be expected of a dendritic constituent. In contrast, the other major bands on the gel were found in all 3 regions of the axon, but not necessarily to the same extent. A prominent $94 \mathrm{kDa}$ band, for example, was sometimes enriched in the ganglion (Fig. 8, inset), but was also clearly present in the periphery (band 19, Fig. 8). We speculate that this constituent may have more than one destination in the cell.

Evidence that the $78 \mathrm{kDa}$ band is made by $\mathrm{R} 2$ and not $\mathrm{R} 1$ derives from experiments in which we selectively killed one of the cells and then examined the polypeptides transported to the pleural ganglion (Fig. 9). The $78 \mathrm{kDa}$ constituent was present when $R 2$ was alive and $R 1$ was dead, but not when $R 2$ had been killed.

\section{Discussion}

Distal dendrites and R2's role in behavior

Our work shows that R2 has 2 dendritic arborizations, one located proximal to the cell body and the other distal, in the pleural ganglion. We use the word "dendrite" for want of a better term, and merely to denote a region that receives chemical synaptic input. R2 synapses on unicellular mucous glands in the skin and constitutes the efferent portion of a circuit that regulates mucus release during certain behaviors (Rayport et al., 1983). Understanding R2's role in the behaviors now requires that we know how the 2 sets of dendrites interact to activate the glands.

The electrophysiological data indicate that the distal dendrites receive synaptic input (Fig. 3) that may lead to spike initiation. The elicited action potentials will propagate in both an orthograde direction, along peripheral axons to the glands, and in a retrograde direction, along the axon in the right connective. The former activity creates an unusual situation in that the presynaptic neuron, dendrites, and peripheral $\mathrm{R} 2$ axons comprise a local circuit for activation of the glands independent of the R2 

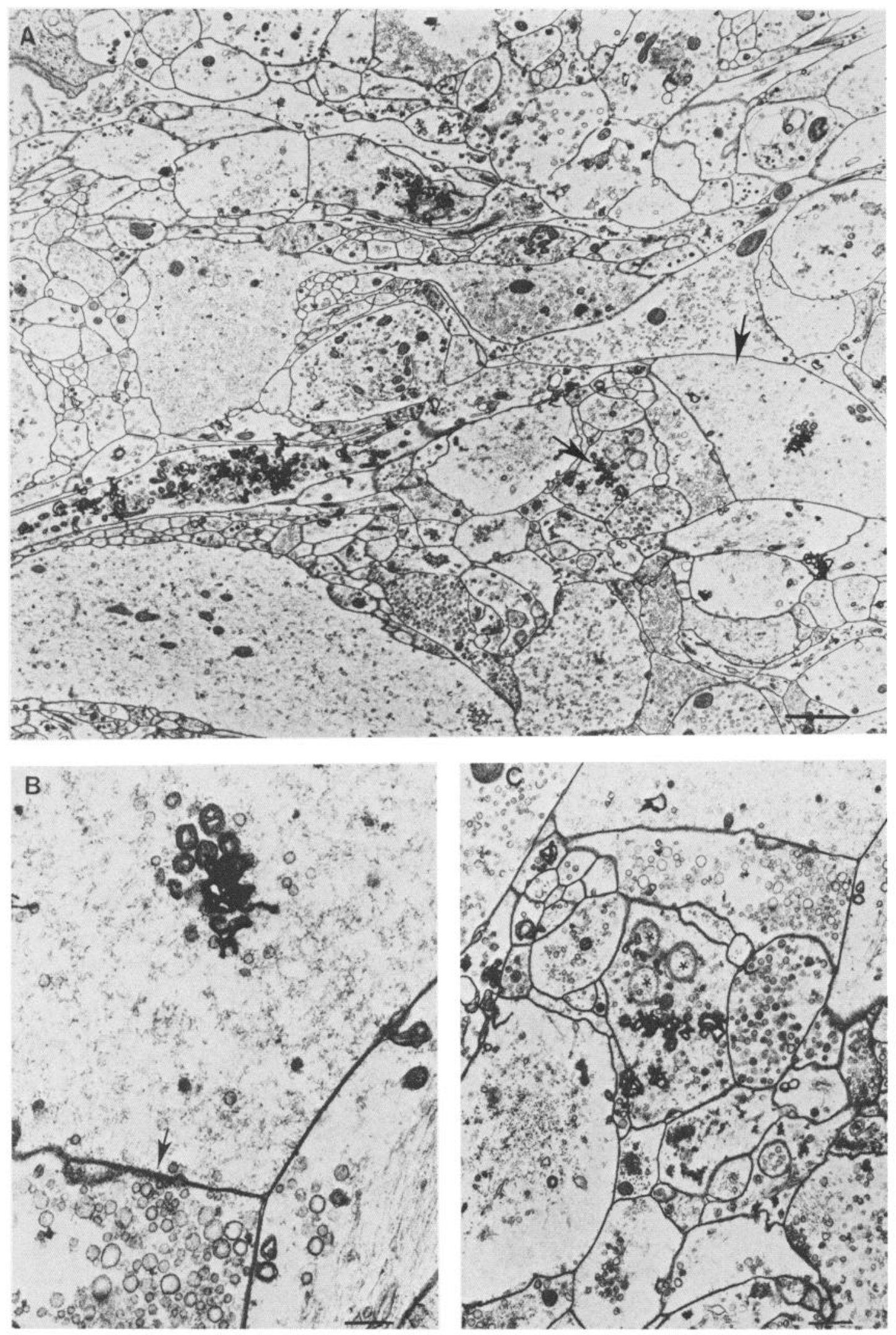

Figure 7. Ultrastructure of R2's dendrites in the neuropil of the pleural ganglion. $A$, Section showing several labeled dendrites in the neuropil. Regions indicated by the arrows are shown at higher magnification in $B$ and $C$. Calibration, $10 \mu \mathrm{m}$. $B$, Putative synapse (arrow) with vesicles abutting a thickened membrane onto a radiolabeled $\mathrm{R} 2$ dendrite. Calibration, $2 \mu \mathrm{m}$. $C$, Unlabeled fingerlike processes of unknown origin projecting into R2's dendrites. Calibration, $2 \mu \mathrm{m}$. 

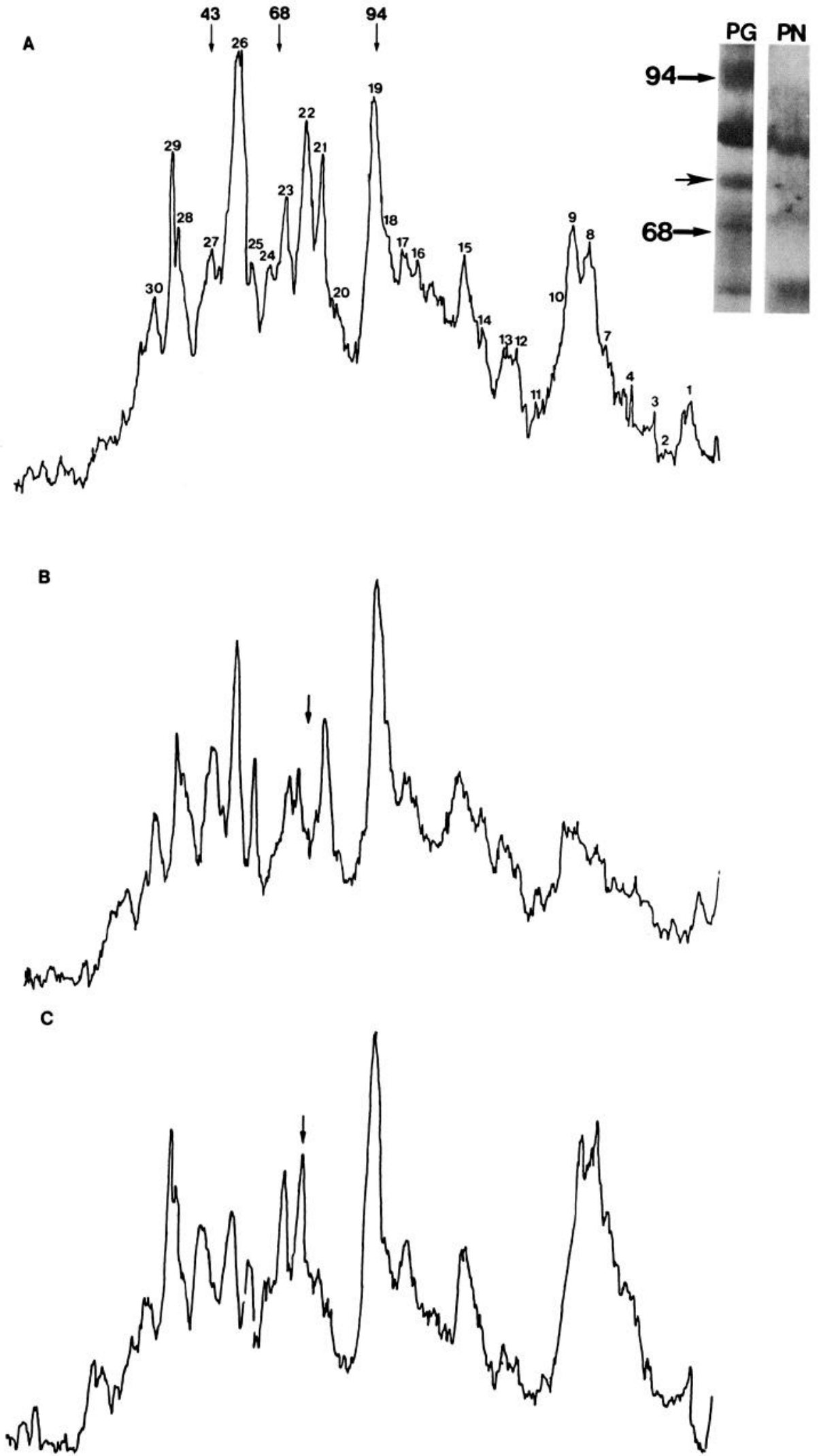

Figure 8. SDS-PAGE of rapidly transported ${ }^{35} S$-methionine-labeled proteins. Fifty hours after exposing the abdominal ganglion to a $5 \mathrm{hr}$ pulse of labeled methionine, the pleural ganglion $(A)$, peripheral nerves $(B)$, and distal one-third of the right connective $(C)$ were separated and their proteins examined by SDS-PAGE. Radiolabeled proteins were detected by fluorography and the autoradioraphs were scanned. A band of $78 \mathrm{kDa}$ is seen in the profiles from the distal connective (arrow) and pleural ganglion (22) that is not present in the peripheral nerves (arrow). Inset, Portion of a fluorograph from another experiment showing the presence of the $78 \mathrm{kDa}$ band (arrow) in the pleural ganglion $(P G)$ and its absence in the peripheral nerves $(P N)$. A major band at the $94 \mathrm{kDa}$ marker is also enriched in the ganglion in this experiment. This is not exclusively a dendritic component, since it is also prominent (peak 19) in the peripheral nerves, as shown in $B$. cell body and axon in the right connective. The retrograde spike, on the other hand, will attenuate or even cancel an orthodromic action potential triggered by stimulation of the proximal dendrites. Thus, simultaneous input to the dendrites will not be additive, but will favor activation of the glands via the distal dendrites. If these ideas are correct, then R2's axon in the right connective should normally propagate action potentials in either direction. In fact, bidirectional spike conduction has been seen 


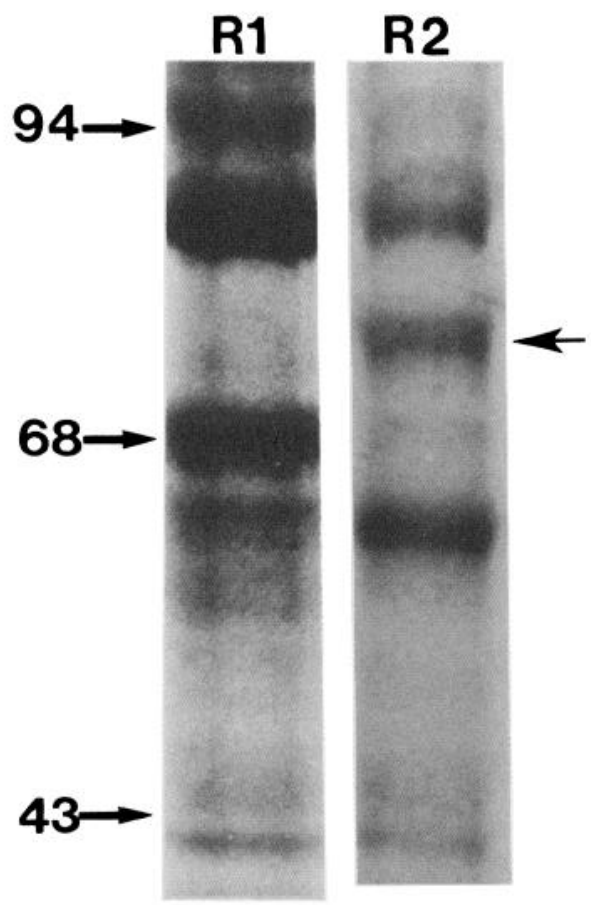

Figure 9. SDS-PAGE of proteins rapidly transported to the pleural ganglion in the presence and absence of R2 or R1. Photograph of a portion of the gel containing radiolabeled proteins from the pleural ganglion, showing the region containing the putative dendritic component. In the gel on the right, R2 was alive, but prior to exposure to ${ }^{35} S$-methionine, R1 was killed by multiple impalements of the soma until there was no resting potential. Note that the $78 \mathrm{kDa}$ band (arrow) is present. In the gel on the left, R2 was killed while R1 was alive: the $78 \mathrm{kDa}$ band is absent.

in R2's axon in freely moving animals (Eberly and Pinsker, 1979).

Another factor that must be considered before R2's role can be appreciated is the identity of the neurons responsible for the inputs. R2 is stimulated by RE sensory cells in the abdominal ganglion, and the input undergoes habituation and facilitation during gill withdrawal (Rayport, 1981). The input to the distal dendrites is not known, but is likely to include neurons found previously by Shimahara and Tauc (1975), or recently found interneurons that mediate the tail-withdrawal reflex (Cleary and Byrne, 1985). Once the presynaptic cells are known, interactions between the various branches of the circuit can be explored to determine their contributions to glandular release.

\section{Regional differentiation of $R 2$}

The existence of the distal postsynaptic arborization requires that there be a difference between the macromolecules of that region and R2's presynaptic terminals in the skin. Both regions, however, receive their proteins by rapid axonal transport from the right connective axon. We examined the transported polypeptides by SDS-PAGE and found a $78 \mathrm{kDa}$ band that has been identified as a dendritic constituent. Several lines of evidence support our contention. First, our electron micrographs show that the dendrites do not contain ribosomes; dendritic constituents, therefore, cannot be synthesized locally. Second, biochemical and autoradiographic analyses show that transported proteins and glycoproteins (Fig. 4 and Ambron et al., 1985) accumulate in R2's dendrites, indicating that they are the final destination for at least some constituents. Consistent with this idea, the $78 \mathrm{kDa}$ material accumulated in the pleural ganglion and was never seen in the peripheral nerves (Fig. 8). This behavior was unique to the $78 \mathrm{kDa}$ band; the other major polypeptides were all transported to the periphery. Finally, the 78 $\mathrm{kDa}$ component must be made by $\mathrm{R} 2$, since it is synthesized and transported only when R2 is alive (Fig. 9).

It was expected that the dendrites would have proteins not present at R2's presynaptic terminals, since these regions are functionally and structurally distinct. We originally considered the possibility that dendritic components might arise by a posttranslational modification of transported proteins. The fact that the $78 \mathrm{kDa}$ material is also present in the distal portions of the $\mathrm{R} 2$ axon in the right connective (Fig. $8 \mathrm{C}$ ), however, argues against this idea. It seems more likely that the dendritic constituents are sorted from the presynaptic proteins destined for the periphery. Where would sorting take place? Given the constraint that macromolecular synthesis is restricted to the neuronal soma, selective transport has already been shown to be one way in which regional distinctions are established. R2's cell body synthesizes hundreds of individual polypeptides in a $24 \mathrm{hr}$ period, but only about 30 are exported into the axon for rapid transport (Ambron et al., 1985; Drake et al., 1985). Likewise, R2 elaborates 9 major membrane glycoproteins, 8 of which enter the axon, while the other is transported to the surface of the cell body (Ambron, 1981).

In this respect, $\mathrm{R} 2$ is analogous to epithelial cells, where the apical and basolateral cell surfaces differ in protein composition (for a review, see Simons and Fuller, 1985), and the liver, where the membrane opposed to a bile canaliculus contains protein species not found elsewhere on the surface (Hubbard et al., 1983; Matsuura et al., 1984). A unique aspect of R2, however, is that there are likely to be 2 sites of sorting. One site, located in the cell soma, would segregate pre- and postsynaptic components from those that will remain in the cell body. The second, at or near the dendrites, would direct postsynaptic components to the dendritic arbor, while allowing the presynaptic constituents to be transported to the periphery. Another possibility is that the dendritic and peripheral constituents are sorted into different vesicle populations in the cell body and that the different vesicles are then transported directly to their destinations. An antibody against the $78 \mathrm{kDa}$ polypeptide would be useful as an immunocytochemical probe to localize the site at which the sorting takes place. Such experiments would also directly address the role of the unusual dendritic openings (Figs. 5, 6) in the sorting process.

It was initially believed that selective transport created differences in composition between the cell body and presynaptic terminals (the R2 axolemma acquires few, if any, of its constituents from these protein pools) (Ambron et al., 1985). Now that we know that the material moving along the axon is further segregated into pre- and postsynaptic components, we can try to identify the signal on the $78 \mathrm{kDa}$ material that distinguishes it from peripherally directed proteins. Rapidly transported proteins pass through the endoplasmic reticulum-Golgi pathway (Hammerschlag et al., 1982). Proteins that traverse these membranes are destined for various domains in the cell, and each must contain a signal that confers information as to its destination. To date, the only signal that has been unequivocally identified is that indicating the presence of mannose-6-phosphate on certain enzymes to be transported to lysosomes (Sly and Fischer, 1982). There is also some evidence that glucose- 
1-phosphate may direct specific glycoproteins to the surface of retinal ganglion cells (Koro and Marchase, 1982). Both signals are posttranslational modifications. It is relevant, therefore, that R2's axon in the right connective has glycosyl transferases capable of adding sugar moieties to proteins exported from R2's cell body (Ambron and Triestman, 1977). Perhaps these reactions are involved in directing vesicles and/or their constituents to pre- or postsynaptic terminals. Work is currently under way to explore this possibility.

\section{References}

Ambron, R. T. (1981) Differences in the distribution of specific glycoproteins among the regions of a single identified neuron. Brain Res. 239: 489-505.

Ambron, R. T., and S. N. Treistman (1977) Glycoproteins are modified in the axon of R2, the giant neuron of Aplysia californica, after intra-axonal injection of ${ }^{3} \mathrm{H}$-galactosamine. Brain Res. 121: 287-309.

Ambron, R. T., J. E. Goldman, E. B. Thompson, and J. H. Schwartz (1974) Synthesis of glycoproteins in a single identified neuron of Aplysia californica. J. Cell Biol. 61: 649-664.

Ambron, R. T., A. A. Sherbany, L. J. Skolnik, and J. H. Schwartz (1979) Distribution of membrane glycoproteins among the organelles of a single identified neuron of Aplysia. 1. Association of a ${ }^{3} \mathrm{H}$-glycoprotein with vesicles. Brain Res. 207: 17-32.

Ambron, R. T., H. S. Den, and S. Schacher (1985) Synaptogenesis by single identified neurons in vitro: Contribution of rapidly transported and newly synthesized proteins. J. Neurosci. 5: 2857-2865.

Bailey, C. H., P. Kandel, and M. Chen (1981) Active zones at Aplysia synapses: Organization of presynaptic dense projections. J. Neurophysiol. 46: 356-368.

Bonner, W. M., and R. A. Lasky (1974) A film detection method for tritium labeled proteins and nucleic acids in polyacrylamide gels. Eur. J. Biochem. 46: 83-88.

Cleary, L. J., and J. H. Byrne (1985) Interactions contributing to the mediation and modulation of the tail withdrawal reflex in Aplysia. Soc. Neurosci. Abstr. 11: 642.

Drake, P. F., M. M. Oblinger, and R. J. Lasek (1985) Synthesis and fast axonal transport of protein in the isolated Aplysia nervous system. Brain Res. 332: 47-57.
Eberly, L., and H. Pinsker (1979) Activity in giant axons affects conduction velocity in intact Aplysia. Tex. Soc. Neurosci. Abstr.

Eisenstadt, M., J. E. Goldman, E. R. Kandel, H. Koike, J. Koester, and J. H. Schwartz (1973) Intrasomatic injection of radioactive precursors for studying transmitter synthesis in identified neurons of Aplysia californica. Proc. Natl. Acad. Sci. USA 70: 3371-3375.

Hammerschlag, R., G. C. Stone, F. A. Bolen, J. D. Lindsey, and M. H. Ellisman (1982) Evidence that all newly synthesized proteins destined for fast axonal transport pass through the Golgi apparatus. J. Cell Biol. 93: 568-575.

Hubbard, A. L., D. A. Wall, and A. Ma (1983) Isolation of rat hepatocyte plasma membranes. 1. Presence of three major domains. J. Cell Biol. 96: 217-229.

Koro, L. A., and D. B. Marchase (1982) A UDP-glucose:glycoprotein glucose-1-phosphotransferase in embryonic chicken neural retina. Cell 31: 739-748.

Laemmli, V. K. (1970) Cleavage of structural proteins during the assembly of the head of bacteriophage T4. Nature 227: 680-685.

Matsuura, S., S. Eto, K. Kato, and Y. Tashino (1984) Ferritin immunoelectron microscopic localization of $5^{\prime}$-nucleotidase on rat liver cell surface. J. Cell Biol. 95: 864-875.

Rayport, S. G. (1981) Development of the functional and plastic capabilities of neurons mediating a defensive behavior in Aplysia. Ph.D. dissertation, Columbia University, NY.

Rayport, S. G., and E. R. Kandel (1980) Developmental modulation of an identified electrical synapse: Functional uncoupling. J. Neurophysiol. 44: 555-567.

Rayport, S. G., R. T. Ambron, and J. Babiarz (1983) Identified neurons $\mathrm{R} 2$ and $\mathrm{LPI}_{1}$ control mucus release in Aplysia. J. Neurophysiol. 49: 864-876.

Shimahara, T., and L. Tauc (1975) Multiple interneuronal afferents to the giant cells in Aplysia. J. Physiol. (Lond.) 247: 299-319.

Simons, K., and S. D. Fuller (1985) Cell surface polarity in epithelia. Annu. Rev. Cell Biol. 1: 243-248.

Sly, W. S., and H. D. Fischer (1982) The phosphomannosyl recognition system for intracellular transport of lysosomal enzymes. J. Cell Biochem. 18: 67-85.

Thompson, E. B., J. H. Schwartz, and E. R. Kandel (1976) A radioautographic analysis in the light and electron microscope of identified Aplysia neurons and their processes after intrasomatic injection of L-3H-fucose. Brain Res. 112: 251-281.

Winlow, W., and E. R. Kandel (1976) The morphology of identified neurons in the abdominal ganglion of Aplysia californica. Brain Res. 112: $221-249$. 